CERN LIBRARIES, GENEVA

\title{
Precision Tests of the Standard Model on the Toponium Resonance
}

B. Grzadkowski and P. Krawczyk

Inst. of Theor. Physics, Warsaw University

J. H. Kühn and R. G. Stuart

CERN -- Geneva

\begin{abstract}
A B S T R A C T
The viability of using toponium for precision tests of the standard model is discussed. It is found that the measurement of the longitudinal polarization asymmetry, $\alpha_{R L}$, to an accuracy of 0.05 can yield $\sin ^{2} \theta_{W}$ to a precision of up to $4 * 10^{-3}$ and is thus sensitive to one-loop electroweak radiative corrections. The leading logarithmic corrections are given.
\end{abstract}


Recently experimental evidence has been presented [1] for a top quark with a mass of about $40 \mathrm{GeV}$. This suggests that toponium might be found in a mass region where strong, electromagnetic and weak decays are of comparable magnitude [2]. The coupling of toponium to lepton pairs receives contributions both from the virtual photon and the $Z$ [fig.1]. Since toponium ${ }^{3} S_{1}$ is by construction a vector particle it is only the vector part of the neutral current coupling to top quarks which contributes at the toponium vertex. This coupling can be determined either through the relative difference in the production cross section for right handed versus left handed polarized beams ${ }^{*}$ )

$$
\frac{\sigma_{R}-\sigma_{L}}{\sigma_{R}+\sigma_{L}}=\alpha_{R L}
$$

through the forward backward asymmetry of $\mu^{-}$from the $\mu$ pair final state

$\frac{d N}{d \cos \theta}=\frac{3}{8}\left(1+\cos ^{2} \theta+\alpha_{F B} 2 \cos \theta\right)(2)$

or through the forward backward asymmetry of leptons from semileptonic decays of a single quark (SQD) in toponium [3].

$$
\frac{d N}{d \cos \theta_{+}}=\left(1+\alpha_{R L} \& \cos \theta_{+}\right)
$$

(3)

$f$ characterizes the analysing power of these leptons. For leptons from quark decay $f_{t}=1$, for leptons from weak decays of a statistical mixture of $T$ and $T^{*} \quad f_{T}=1 / 2$. Leptons from SQD's originate with equal probability from each of these sources and thus

$$
\xi=\left(f_{t}+f_{T}\right) / 2
$$

This prediction can be tested independently, by studying leptonantilepton angular correlations from double-semileptonic decays

$$
\frac{d N}{d \cos \theta_{+-}}=\left(1-f_{t} \phi_{T} \frac{1}{3} \cos \theta_{+-}\right)
$$

(5)

*) Here and in the following we ignore interference between the continuum and the resonance which is relevant only for $\left|m_{Z}-m_{V}\right|<2 r_{Z}$. 
Since $\alpha_{F B}$ is related to $\alpha_{R L}$ by the simple relation,

$$
\alpha_{F B}=\alpha_{R L}^{2}
$$

all measurements can be interpreted as a determination of $\alpha_{R L}$. The corrections are independent of the quarkonium wave function and thus could serve to determine the weak coupling of top quarks.

It can be shown [4] that $\alpha_{R L}$ can be determined to an accuracy better than 0.10 easily with polarized beams. However, also with unpolarized beams such an accuracy can be achieved at LEP with modest demands for integrated luminosity $\left(\int d t \mathscr{L} \cong 20 \mathrm{pb}^{-1}\right)$, and a reduction of the statistical error by even another factor two to three seems feasible after one year of measurements at the $1 \mathrm{~S}$ or $2 \mathrm{~S}$ resonance $^{*}$ ).

In this note we shall show that such a measurement would not only lead to a verification of the weak isospin of the top quark, but could even serve as an independent test of the higher order corrections within the standard or other models. To illustrate this point, we shall first compare the asymmetry, as calculated in lowest order and through formulae, which take the leading log corrections into account. The difference between the two predictions may be as large as 0.25 for some toponium masses. In a second step we shall then demonstrate that an accuracy of 0.05 in the determination of $\alpha_{R L}$ would correspond to an accuracy of up to $4 * 10^{-3}$ in the determination of $\sin ^{2} \theta_{W}$.

At the time when toponium physics will be studied at LEP, the most accurate parameters which fix the standard model will be $\alpha, G_{\mu}$ and $m_{Z}$. Using only lowest order relations, the asymmetry $\alpha_{R L}$ is given through

$$
\alpha_{R L}=-2 \frac{\operatorname{Re}_{2}\left(\lambda^{*} \lambda^{\prime}\right)}{|\lambda|^{2}+\left|\lambda^{\prime}\right|^{2}}
$$

where

$$
\lambda=\frac{-e Q_{t}}{m_{v}^{2}}+\frac{g^{2}}{16 \cos ^{2} \theta_{w}} \frac{v_{e} v_{t}}{m_{v}^{2}-m_{z}^{2}+i m_{z} \Gamma_{z}}\left(g_{a}\right)
$$

*) Note that for toponium below $100 \mathrm{GeV} \sigma * \mathrm{~B}$ (SQD) is approximately the same for the $1 \mathrm{~S}$ and $2 \mathrm{~S}$ states, and the two samples could be simply combined. 
$-3-$

and

$$
\lambda^{\prime}=\frac{g^{2}}{16 \cos ^{2} \theta_{w}} \frac{a_{e} v_{t}}{m_{v}^{2}-m_{z}^{2}+i m_{z} \Gamma_{z}}
$$

The neutral current coupling is given by

$$
\begin{aligned}
& \frac{g^{2}}{16 \cos ^{2} \theta_{w}}=\frac{G_{\omega} m_{z}^{2}}{2 \sqrt{2}} \\
& v_{f}=\left(2 I_{3}-4 Q_{f}^{2} \sin ^{2} \theta\right) ; \quad a_{f}=2 I_{f}
\end{aligned}
$$

and the Weinberg angle is calculated through

$$
\sin ^{2} \theta_{w}=\frac{1}{2}\left(1-\sqrt{1-\frac{2 \sqrt{2} \pi \alpha}{G_{\omega} m_{z}^{2}}}\right)
$$

The resulting prediction for $\alpha_{R L}$ is shown in fig. 2 as a function of $m_{V}$, for $m_{Z}$ fixed to $94 \mathrm{GeV}$.

The leading first order corrections are proportional to $\alpha \ln \left(m_{V}{ }^{2} / m_{f}{ }^{2}\right)$ and are easily evaluated $\left.{ }^{*}\right)$. We define

$$
\cos \theta_{w} \equiv m_{w} / m_{z}
$$

and use standard methods [6] to calculate $m_{W}$ and thus $\sin ^{2} \theta_{W}$ from our input parameters. The leading log result is simply given by

$$
\sin ^{2} \theta_{w}=\frac{1}{2}\left(1-\sqrt{1-\frac{2 \sqrt{2} \pi \alpha}{G_{\mu} m_{z}^{2}\left(1-\Delta_{\alpha}\right)}}\right)
$$

and

$$
\Delta_{\alpha}=\frac{\alpha}{3 \pi} \sum_{f} Q_{f}^{2} \ln \frac{m_{v}^{2}}{m_{f}^{2}} \approx 0.07
$$

Using this result for the Weinberg angle, the relation between the neutral current coupling $\left(g / 4 \cos \theta_{W}\right)^{2}$ and $G_{\mu} m_{Z}{ }^{2 / 2} \sqrt{2}$ as given in eq. (10) remains unaffected by leading log corrections. The form of the neutral current contribution to the amplitude, as given in eq. (9), remains thus unchanged.

${ }^{*)}$ For a pedagogical discussion see e. g. [5]. 
The leading effect of higher order corrections on the electromagnetic contribution can be absorbed in an increase of the electromagnetic amplitude by the factor $\left(1-\Delta_{\alpha}\right)^{-1}$. The ratio between neutral current and electromagnetic contribution to $\lambda$ is now given by

$$
\frac{\lambda(n c)}{\lambda(\mathrm{em})}=\frac{v_{e} v_{t}}{\left(4 \sin \theta_{w} \cos \theta_{w}\right)^{2}} \frac{m_{v}^{2}}{m_{v}^{2}-m_{z}^{2}+i m_{z} \Gamma_{z}}(15)
$$

and the polarization asymmetry is again given by eq. (8), with *)

$$
\begin{aligned}
& \lambda=e^{2}\left(1-\Delta_{\alpha}\right)^{-1}\left(-\frac{Q_{t}}{m_{v}^{2}}+\frac{v_{e} v_{t}}{\left(4 \sin \theta_{w} \cos \theta_{w}\right)^{2}} \frac{1}{m_{v}^{2}-m_{z}^{2}+i m \Gamma_{z}}\right. \\
& \lambda^{\prime}=e^{2}\left(1-\Delta_{\alpha}\right)^{-1} \frac{a_{e} v_{t}}{\left(4 \sin \theta_{w} \cos \theta_{w}\right)^{2}} \frac{1}{m_{v}^{2}-m_{z}^{2}+i m_{z} \Gamma_{z}}
\end{aligned}
$$

In fig. 2 we compare the prediction from $\alpha_{R L}$ based on this second leading log improved formulae to the lowest order result. For some of the masses the difference between the two predictions may amount up to 0.25 and thus these corrections could be tested experimentally. Evidently our result is unaffected by QCD corrections to the toponium-y or toponium- $Z$ coupling, which modify lowest order and leading log correction in the same way.

The measurement of $\alpha_{R L}$ could also serve for an independent determination of $\sin ^{2} \theta_{W}$ and thus could lead to an independent verification of higher order corrections to the standard model. To evaluate the sensitivity of this measurement, we consider $m_{Z}$ and $\alpha_{R L}$ to be fixed and use eqs. (8) and (16) to solve for $\sin ^{2} \theta_{W}$. The relative error in $\sin ^{2} \theta_{W}$ is determined by the relative error in $\Delta \alpha_{R L}$ through

$$
\Delta \sin ^{2} \theta_{w}=\left(\frac{d \alpha_{R L}}{d \sin ^{2} \theta_{w}}\right)^{-1} \Delta \alpha_{R L}
$$

$d \alpha_{R L} / d\left(\sin ^{2} \theta_{W}\right)$ is shown as a function of $m_{V}$ in fig.2. Its magnitude varies between zero and a value as high as 12 (for $m_{V}=92 \mathrm{GeV}$ ). Taking a value of 10 for $d \alpha_{R L} / d\left(\sin ^{2} \theta_{W}\right)$ as an illustrative example, an

*) The ratio $\lambda / \lambda^{\prime}$ which is only relevant for the asummetry, coincides with the one of ref.[2]. 
error of 0.05 in $\Delta \alpha_{R L}$ would result in a precision of 0.005 for $\sin ^{2} \theta_{W}$. The additional error in $\sin ^{2} \theta_{W}$ resulting from the uncertainty in the measurement of the toponium mass of about $100 \mathrm{MeV}$ is far smaller. An accuracy of 0.005 cannot compete with a determination of $\sin ^{2} \theta_{W}$ through the $Z$ mass. Although it is also somewhat inferior to the test of the standard model through the forward backward asymmetry on top of the $Z$, it nevertheless is a new process and qualitatively different from the reactions which involve only light fermions in the initial and final states. Here the fermion-Higgs coupling enters the evaluation of the complete one loop corrections in an essential manner [7]. 


\section{REFERENCES}

[1] UA1 Collaboration, B. Arnison et al., Phys. Lett. 147B (1984) 493

[2] For a recent discussion and further references see S. Güsken, J. H. Kühn and P. M. Zerwas, SLAC-PUB-3580/ Cern-TH 4125/85 to be published in Nucl. Phys.

[3] J. H. Kühn and K. H. Streng, Nucl. Phys. B198 (1980) 71

[4] W. Buchmüller et al., LEP study group on toponium physics

[5] G. Altarelli, Internationale Universitätswochen fur Kernphysik (1982), Acta Physica Austriaca, Suppl. 24, 229

[6] A. Sirlin, Phys. Rev. D22 (1980) 971

[7] B. Grzadkowski, P. Krawczyk, J. H. Kühn and R. G. Stuart, in preparation

\section{FIGURE CAPTIONS}

Figure 1: $\quad$ Lowest order contributions to toponium production in $\mathrm{e}^{+}-\mathrm{e}^{-}$-annihilation.

Figure 2: $\quad$ Lowest order and leading log corrected prediction for the polarisation asymmetry on the toponium resonance, and its sensitivity towards changes of $\sin ^{2} \theta_{W}$. 


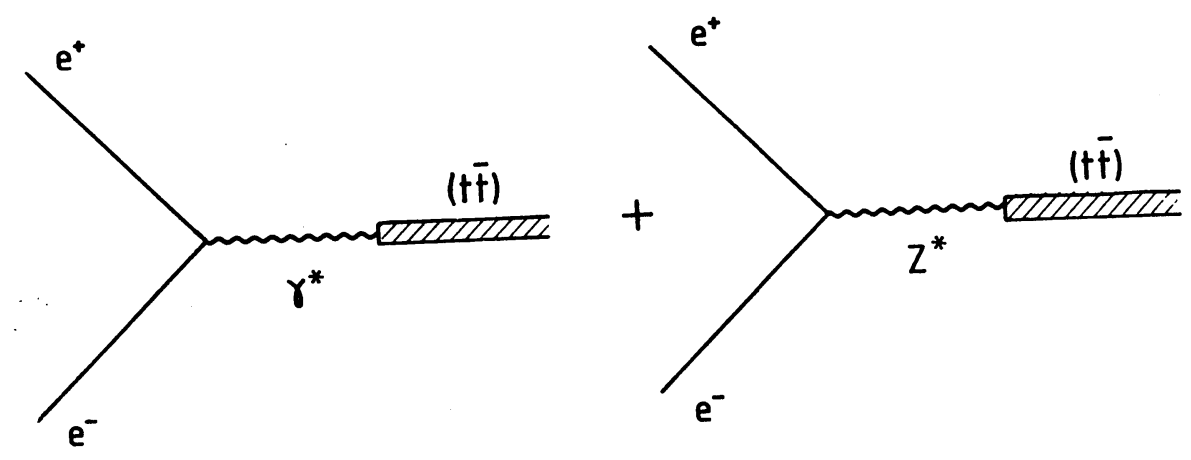

- Figure 1 - 


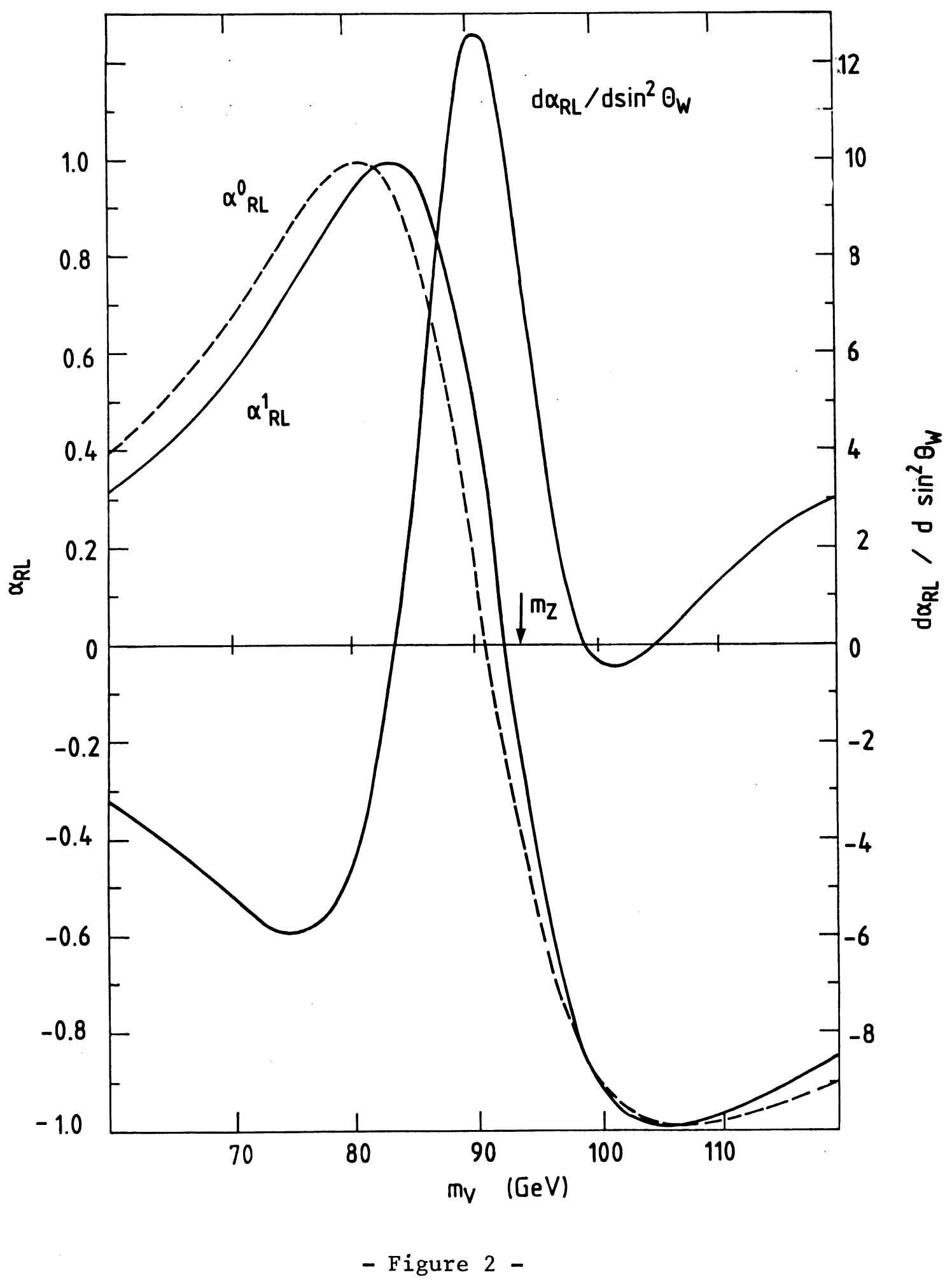

